\title{
Robust Blind Digital Watermarking in Contourlet Domain
}

\author{
Sreejith.V \\ Department of Electronics and \\ Communication, \\ FISAT, \\ MG University,India
}

\author{
Srijith.K \\ Assistant Professor \\ Department of Electronics and \\ Communication,FISAT, \\ MG University,India
}

\author{
Rajesh Cherian Roy \\ Associate Professor \\ Department of Electronics \\ and Communication,FISAT, \\ MG University, India
}

\begin{abstract}
This paper presents an invisible robust blind digital watermarking algorithm using adaptive and distributed quantization in contourlet domain for colour images. In the proposed watermarking scheme, the blue plane of the colour image is decomposed into series of multiscale, local and directional sub images using contourlet transform. The low frequency component of the transform is divided into non overlapping blocks, into which Arnold scrambled binary watermark is embedded, with help of adaptive quantization step value of each block. A blind detection algorithm is employed for the watermark recovery process, which does not require the original image. Experimental results show that the proposed algorithm is highly resistant to attacks such as JPEG compression, noising, cropping etc. The proposed method is compared with an odd-even quantization based technique and the results prove that the adaptive quantization based technique gives better robustness, under similar conditions.
\end{abstract}

\section{General Terms}

Industrial application, Security, Watermarking.

\section{Keywords}

Blind digital watermarking, Contourlet transform, Arnold transform, Quantization.

\section{INTRODUCTION}

The rapid development of Internet and media sharing services has introduced a new set of challenging security problems for the distribution of multimedia data such as images, audio and video. Piracy of such data is the most prominent issue in the multimedia world, Digital watermarking has been proposed as a viable solution to illegal copying or reproduction of digital data. The watermarking system as compared to other copy protection system is more advantageous because the watermark is enduringly embedded in the digital contents with a little compromise on the fidelity of multimedia data. The applications like copyright protection and authentication may mostly require the content owner or the authorized buyer to prove the authenticity without reference to the cover work. This creates a demand on blind watermarking techniques over non-blind watermarking techniques. There are a few methods of watermarking which do not use the original data for detecting or recovering the digital watermark embedded into the cover work [1].

Based on the embedding domain, digital watermarking techniques are classified into (i) spatial domain (ii) frequency domain and (iii) spatio-frequency domain. In spatial domain method, the watermark is directly embedded into the host image by changing its pixel value (eg. LSB method) [2]. In the transform domain watermarking schemes, filters are used for the domain transformation and the watermark is spread all over the image. Hence transformed watermarking schemes are more robust compared to spatial domain ones. Transform domain techniques includes Discrete Cosine Transform (DCT), Discrete Fourier Transform (DFT), Discrete Wavelet Transform (DWT) etc [2].

DWT is broadly used for digital watermarking since it has better performance than other transform domain watermarking techniques. DWT is a multiresolution and time frequency representation. It captures only the discontinuities at edge points of the image in three directions at each resolution and lacks in capturing smoothness along the contours. This problem has been solved by the Contourlet Transform (CT) introduced by Do and Vetterli [3, 4]. CT has anisotropy feature and multiple directionality in each resolution, thus it is used for the smooth contour representation of natural images. The basis function of contourlet has different aspect ratio, hence it can capture discontinuity at the edges of the image very easily. These features of CT makes it a good tool for image domain transformation[5].

An algorithm proposed by J.Wei and S.Yong [6], embeds the binary watermark in the low frequency coefficients of the original image using odd even quantization. In this approach, each binary pixel of watermark is embedded into a single coefficient. If any one of the coefficient gets changed due to some attack, the watermark may be lost. To overcome this difficulty, this paper proposes a method in which the watermark is distributed to a coefficient block, which provides resistance to image processing attacks.

\subsection{Arnold transform}

In order to improve the watermarking safety and visual quality of the extracted watermarks, a scrambling operation is done on the original watermarks. Thus the error pixels will not be together if error occurs for one or some regions of watermarking image, but scatter throughout the whole image space when extracted. Considering the simplicity of Arnold transform [7], it is adopted in this paper. Arnold transform (also called Cat mapping) is a clipping transform presented by V. J. Arnold. After applying a specific iteration of Arnold transform to the watermarking image, the locations of pixels are rearranged and it becomes chaotic.
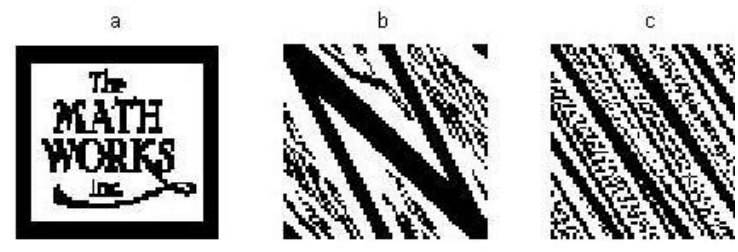

Fig.1. (a) The original digital watermark, and its Arnold transform after (b) 1-iteration, (c) 2-iterations 
The Arnold transform can be defined in the following form:

$$
\left[\begin{array}{l}
i^{\prime} \\
j^{\prime}
\end{array}\right]=\left[\begin{array}{ll}
1 & 1 \\
1 & 2
\end{array}\right] \times\left[\begin{array}{l}
i \\
j
\end{array}\right](\bmod N)
$$

where $(i, j)$ and $\left(i, j^{\prime}\right)$ represent the pixel coordinate of watermark before and after executing Arnold transform, and $N$ is the order of digital watermarking image. The operator "mod" represents the modulo operator. Fig. 1 shows the Arnold transformation of a binary image 'logo', which is used as the watermark.

The inverse Arnold transform is given by:

$$
\left[\begin{array}{l}
i \\
j
\end{array}\right]=\left[\begin{array}{cc}
2 & -1 \\
-1 & 1
\end{array}\right] \times\left[\begin{array}{l}
i^{\prime} \\
j^{\prime}
\end{array}\right](\bmod N)
$$

\subsection{Contourlet Transform}

The Contourlet Transform is a new image geometric transformation technique which is an extension of wavelet transform. It provides a flexible multi resolution, local and directional image expansion. The contourlet transform is realized efficiently via a double iterated filter bank structure, which consists of Laplacian Pyramid (LP) and Directional Filter Bank (DFB) Structure [8,9,10]. Hence it is also known as Pyramidal Directional Filter Bank (PDFB). The Laplacian Pyramid filter produce a low frequency and a band pass image, band pass images represents the discontinuities at the edges of the image. Directional Filter Bank structure link these point discontinuities into smooth contours at different orientation. This filter bank produces a multiresolution, multi directional image with smooth contour, thus it is known as contourlet transform.

Fig. 2 describes the Contourlet decomposition, in which the lowpass image and bandpass image are generated by LP decomposition at each level. The band pass image is then fed into DFB which captures the directional information of the image. Using DFB, each LP bandpass image is decomposed into a number of wedge shaped subbands. The $m$-level DFB decomposes the bandpass image into $2^{\mathrm{m}}$ directional subbands.

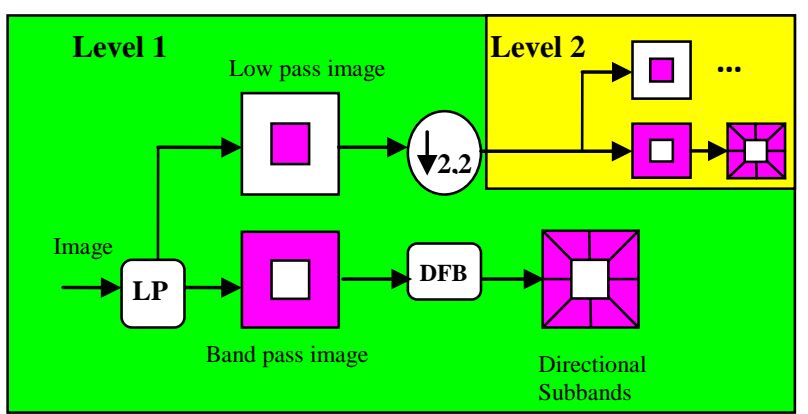

Fig.2. The diagram of the Contourlet transform

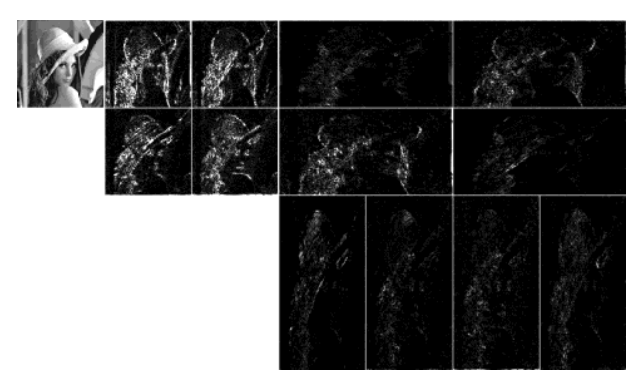

\section{Fig.3 Two-level Countourlet decomposition}

The Contourlet Transform of 'Lena' image is shown in Fig.3. It is a two level transformation with 8 and 4 directional subbands in level 1 and 2 respectively. Contourlet Transform provides the feature of specifying the number of directional decomposition at every level of multi resolution pyramid. It performs better than wavelet transform in capturing directional information at edge points as well as contours of the image.

The rest of the paper is organized as follows. Section 2 describes the proposed scheme which involves a watermark embedding process and a watermark recovery procedure. Section 3 presents the simulation results. The conclusions are presented in Section 4.

\section{ALGORITHM}

\subsection{Watermark Embedding process}

The watermark embedding process is as follows:

1) Perform Arnold transform to the watermark binary image $(W)$, using a scrambling key ' $K$ '.

2) Select Blue-plane (I) of the cover image, then perform two level contourlet transform of the image $I$. The lowfrequency component $I_{L}$ is the embedding domain.

3) Divide the embedding domain $I_{L}$ into non overlapping blocks $A i$ of size ' $n \times n$ ', where $i=1,2,3 \ldots M . M$ is the total number of pixels in the watermark image.

$$
n=\operatorname{floor}\left(\frac{N 1}{N 2}\right)
$$

$\mathrm{N} 1$ is the row size of low-frequency component and $\mathrm{N} 2$ is the row size of the watermark.

4) Calculate adaptive quantization value of each block using the following equations.

Average pixel value in a block is

$$
P i=\sum_{x=1}^{n} \sum_{y=1}^{n} A i(x, y)
$$

For each block $A i$ the adaptive quantization step value $\delta_{i}$ is computed as follows

$$
\delta i=\frac{F \operatorname{loor}(\log 2(P i) * 1000)}{1000}
$$

$\Delta i=\delta i+\Delta$ 
Where $\Delta$ is the quantization step value.

5) For each block following values are calculated.[11]

$S i b=\|A i\|+1$

where $\|\cdot\|$ represents the Euclidean norm

$S i=f l o o r\left(\frac{S i b}{\Delta i}\right)$

$\hat{S} i a= \begin{cases}(S i+1.5) \Delta i & \text { if } \bmod (S i, 2)=w i \\ (S i+0.5) \Delta i & \text { if } \bmod (S i, 2) \neq w i\end{cases}$

where $w i$ is the watermark .

Watermarked block is given by

$\hat{\mathrm{A}} i=A i\left(\frac{\hat{\mathrm{S} i a}}{\mathrm{Sib}}\right)$

6) Reconstruct the low frequency component $\hat{I}_{L}$ using $\hat{A} i$, then perform two corresponding inverse Contourlet transform. Using this modified blue plane $(\hat{I})$ watermarked colour image is created.

\subsection{Watermark Extraction}

1) Select the Blue plane $(\hat{I} r)$ of the watermarked image. Perform two level contourlet transform and select the low frequency component $\left(\hat{I} r_{L}\right)$ of the transform.

2) Divide the Ir $r$ into non overlapping blocks Ari of size ' $n$ $x n$ ', where $\mathrm{i}=1,2,3 \ldots M . M$ is the number of pixels in the watermark image.

3) Calculate adaptive quantization value of each block using the following equations.

Average pixel value in a block is

$$
\operatorname{Pri}=\sum_{x=1}^{n} \sum_{y=1}^{n} \operatorname{Ari}(x, y)
$$

For each block Ari the adaptive quantization step value $\delta r i$ is computed as follows

$$
\begin{aligned}
\delta r i & =\frac{F \operatorname{loor}(\log 2(\text { Pri }) * 1000)}{1000} \\
\Delta r i & =\delta r i+\Delta
\end{aligned}
$$

where $\Delta$ is the quantization step value.

$\mathrm{Sr} i$ is calculated using the following equation

$$
\begin{aligned}
& \text { Sri }=\text { floor }\left(\frac{\|\operatorname{Ari}\|+1}{\Delta r i}\right) \\
& w r=\left\{\begin{array}{l}
1 \text { if } \bmod (\operatorname{Sri}, 2)=0 \\
0 \quad \text { if } \bmod (\operatorname{Sri}, 2)=1
\end{array}\right.
\end{aligned}
$$

Where $w r$ is the extracted watermark

4) Perform Arnold anti-scrambling on $w r$ with the scrambling key $K$, and the result $W$ ' is the extracted watermark.

\section{SIMULATION RESULTS}

For the evaluation of the proposed algorithm, a colour 'Lena' image of size (512×512), with depth of $24 \mathrm{bit} / \mathrm{pixel}$ and a binary image 'logo' of size $(64 \times 64)$ is used for the cover image and watermark respectively. In Contourlet transform a "9-7"pyramid filter for LP, and "pkva" orientation filter for DFB are used. The simulation is performed in Matlab 7.1 version. The value of $\Delta$ is set to 50 for all experiments.

Peak-signal-to-noise ratio (PSNR) and structural similarity (SSIM) [12] are used to measure the quality of embedded cover image. In addition, Normalized Correlation (NC) is used for the quality measurement of extracted watermark. In this proposed method, only the values of blue plane are changed. Thus the PSNR of watermarked colour image will be infinity because the MSE of red and green plane is zero. Then for comparison, watermarked blue plane PSNR [13] is calculated using the following equation.

$$
\begin{aligned}
& P S N R=10 \log _{10}\left(\frac{255^{2}}{M S E}\right) \\
& M S E=\frac{1}{M \times N} \sum_{i=1}^{M} \sum_{j=1}^{N}(I(i, j)-\hat{I}(i, j))^{2}
\end{aligned}
$$

$I$ is the blue plane of the cover image and $\hat{I}$ is the watermarked blue plane. $(M, N)$ are size of the image. MSE is the mean square error of the watermarked blue plane.

The second measure used in this paper is structural similarity measure (SSIM) index, which is a region-based numerical metric that places more emphasis on the HVS than PSNR. Mathematically, for images $(I, \hat{I})$, it is expressed as

$$
\operatorname{SSIM}(I, \hat{I})=\frac{\left(\mu_{\hat{I}}+C 1\right)\left(\sigma_{I I}+C 2\right)}{\left(\mu_{I}^{2}+\mu_{I}^{2}+c 1\right)\left(\sigma_{I}^{2}+\sigma_{I}^{2}+c 2\right)}
$$

where $\mu_{I}$ and $\mu_{\hat{I}}$ are the means and $\sigma_{I}^{2}$ and $\sigma_{\hat{I}}^{2}$ are the variances of image $I$ and $\hat{I}$ respectively, c1 and c2 are constants.c1 $=\left(\mathrm{k}_{1} \mathrm{~L}\right)^{2}, \mathrm{c} 2=\left(\mathrm{k}_{2} \mathrm{~L}\right)^{2}$ two variables to stabilize the division with weak denominator, $\mathrm{L}$ is dynamic range of the pixel-values. $\sigma_{I \hat{l}}$ is the covariance of $I$ and $\hat{I}$. Above indicators are used for quantifying the similarity between original data and recovered data.

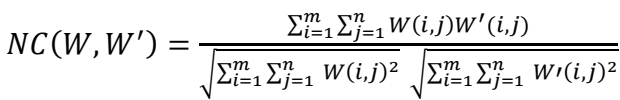

$W$ is watermark $W^{\prime}$ is the retrieved watermark $(\mathrm{m}, \mathrm{n})$ are size of the watermark.

Fig. 4 shows the original cover image, blue plane of the cover image, watermarked blue plane and the watermarked image, when it is not attacked. The value of PSNR is $32.9737 \mathrm{~dB}$ and SSIM is $0.9627(\mathrm{~K}=10)$. This indicates that after embedding the watermark; the distortion of the image is small. 


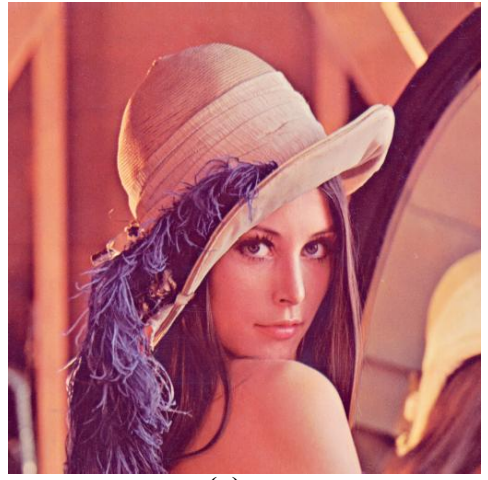

(a)

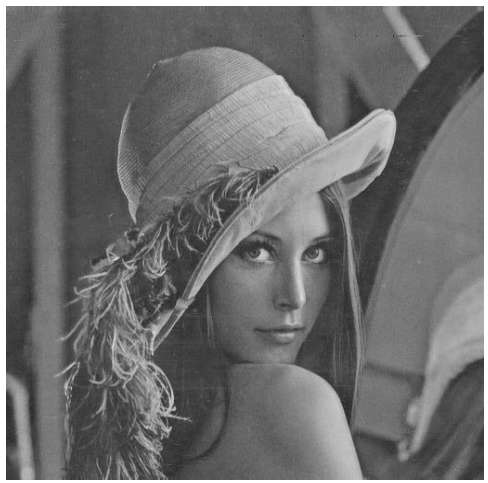

(b)

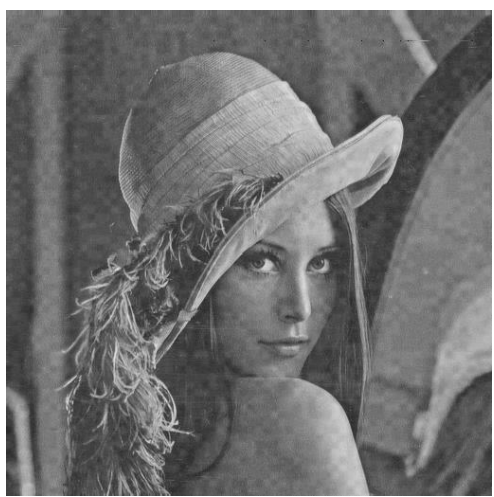

(c)

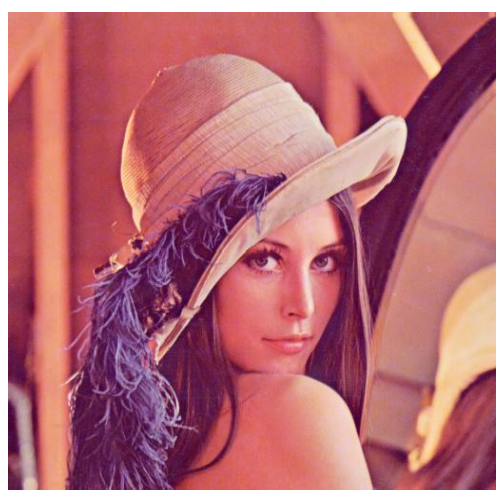

(d)

Fig.4. (a) cover image (b) blue plane (c) watermarked blue plane (d) watermarked image

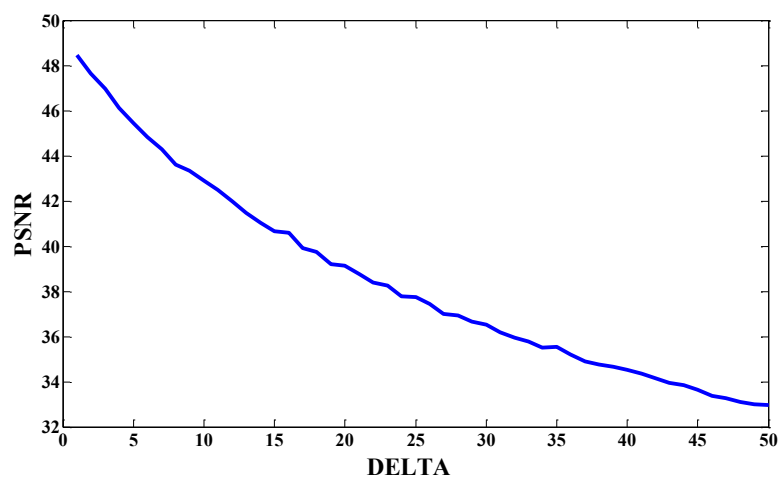

Fig.5. The relationship between PSNR and the step size of the quantization $($ DELTA $=\Delta)$

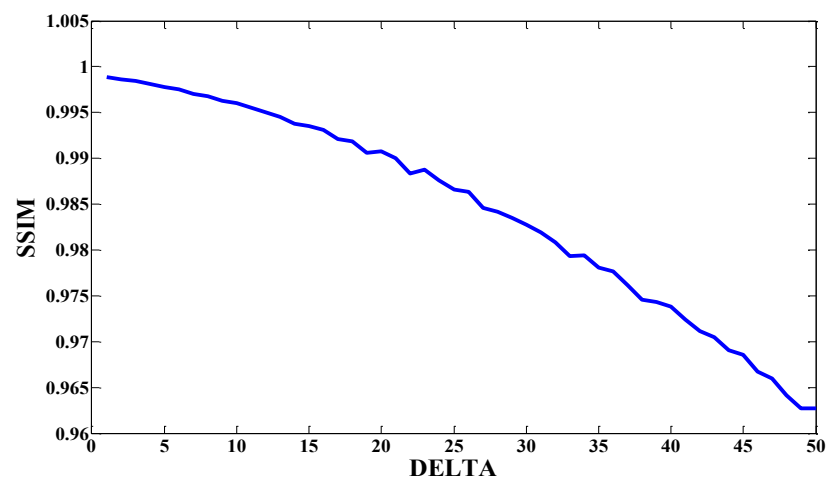

Fig.6. The relationship between SSIM and the step size of the quantization $($ DELTA $=\Delta)$

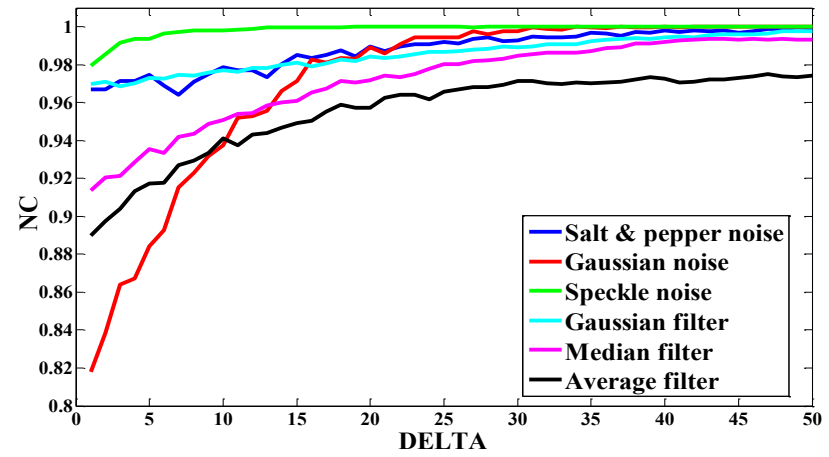

Fig.7. The relationship between NC and the step size of the quantization $($ DELTA $=\Delta$ ) for different attacks.

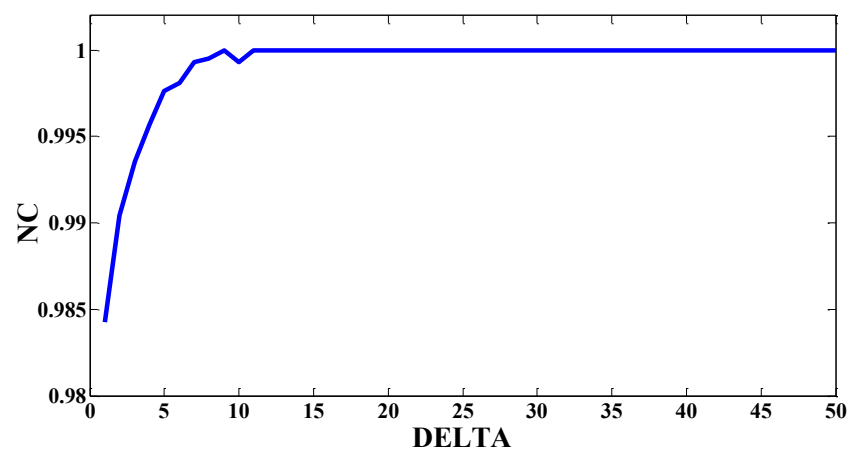

Fig.8. The relationship between $\mathrm{NC}$ and the step size of the quantization $($ DELTA $=\Delta$ ) of .JPEG compressed image. 
Table I. Comparison between proposed algorithm and Reference [6]

\begin{tabular}{|c|c|c|}
\hline Noise & $\begin{array}{l}\text { Proposed } \\
\text { Method }\end{array}$ & Reference [6] \\
\hline $\begin{array}{l}\text { Salt \& pepper noise } \\
\qquad(0.1 \%)\end{array}$ & 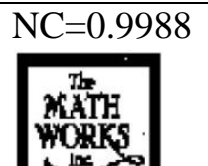 & $\begin{array}{l}\mathrm{NC}=0.9876 \\
\text { ThK } \\
\text { Whis }\end{array}$ \\
\hline Gaussian noise $(0.1 \%)$ & $\begin{array}{l}\mathrm{NC}=0.9998 \\
\text { Whith } \\
\text { Woxks }\end{array}$ & 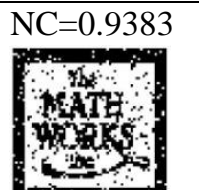 \\
\hline Speckle noise $(0.1 \%)$ & 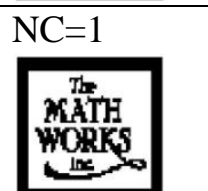 & 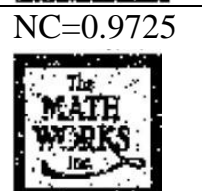 \\
\hline Gaussian filter (3x3) & $\begin{array}{l}\mathrm{NC}=0.9976 \\
\text { Naith } \\
\text { Woxk }\end{array}$ & 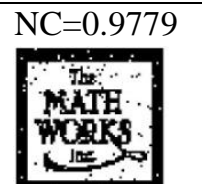 \\
\hline JPEG compression[14] & $\begin{array}{l}\mathrm{NC}=1 \\
\text { Math } \\
\text { WORK }\end{array}$ & 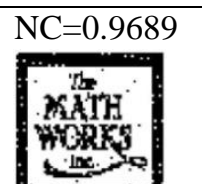 \\
\hline $\begin{array}{l}\text { Median Filter }(3 \times 3) \\
{[15]}\end{array}$ & $\begin{array}{l}\mathrm{NC}=0.9995 \\
\text { Math } \\
\text { Woxks }\end{array}$ & 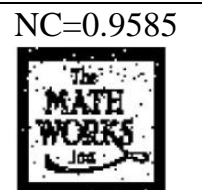 \\
\hline Averaging filter & 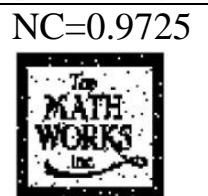 & 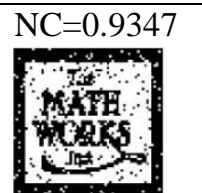 \\
\hline Cropping(1/8) & 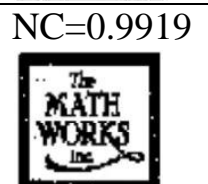 & 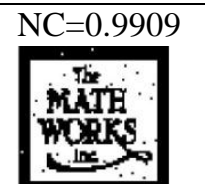 \\
\hline Cropping(1/4) & 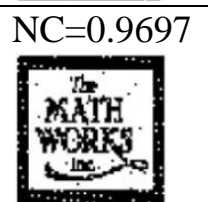 & 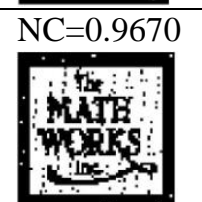 \\
\hline Cropping(1/2) & 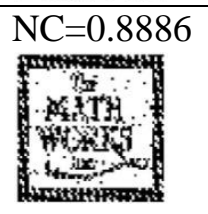 & $\begin{array}{l}\mathrm{NC}=0.8668 \\
4 \\
4\end{array}$ \\
\hline
\end{tabular}

Fig.5 and Fig.6 show that PSNR and SSIM decreases as the quantization value increases.

Plots of NC versus quantization value for different types of attacks are shown in Fig.7 and Fig.8. NC increases with quantization value, i.e. robustness increases. There is a tradeoff between PSNR and NC for the selection of quantization value $(\Delta)$. From figures Fig.7 and Fig.8, the value of $\mathrm{NC}$ is almost 1 for all kind of attacks, for the quantization value of 50 . Hence a quantization value of 50 is chosen for all experiments.

Table I shows the NC of the proposed method with 'lena' as the cover image and 'logo' as the watermark image $(\mathrm{K}=10)$, and the results are compared with [6]. It is seen that the proposed algorithm provides good invisibility and robustness.

\section{CONCLUSION}

This paper proposes a contourlet domain based robust blind digital watermarking algorithm using Arnold transformation and adaptive quantization. An additional quantization value is used for the embedding process, hence increasing the robustness. In addition, some factors such as Arnold scrambling key $(\mathrm{K})$, the size of cover image and watermark image determine the security of the algorithm. Table I clearly shows that the results obtained by the proposed method are better than those obtained in [6]. If the quantization step size is increased, the PSNR will reduce. Future work is aimed at optimizing the method to overcome this problem.

\section{REFERENCES}

[1] Smitha Rao M.S, Jyothsna A.N, Pinaka Pani.R "Digital Watermarking: Applications, Techniques and Attacks" International Journal of Computer Applications (0975 8887) Volume 44- No.7, April 2012.

[2] Darshana Mistry, "Comparison of Digital Water Marking methods"IJCSE Vol. 02, No. 09, 2010, 2905-2909

[3] M.Do, M.Vetterli, "Contourlets : a directional multi resolution image representation" IEEE International Conference on Image Processing (ICIP'2002), vol.1, Rochester, 2002: 357-360.

[4] Do, M.N., Vetterli, M. "The contourlet transform: an efficient directional multiresolution image representation". IEEE trans. Image Processing 2005, 14(2):2091 - 2106

[5] R. Javidan, M. A. Masnadi-Shirazi, Z. Azimifar and M. H. Sadreddini,"A Comparative study between wavelet and Contourlet Transorm Features for Textural Image Classification", Information and Communication Technologies: From Theory to Applications, pp. 1-5, 7 11 April 2008.

[6] Jingjing Wei, Shihua Yong, Xiaohu Ma, "Blind Digital Watermarking Algorithm based on Quantization in Contourlet Domain" 2nd International Conference on eBusiness and Information System Security (EBISS), 2010.

[7] C Wang, J Wang, M Zhou,G Chen,Deyi Li "ATBaM: An Arnold Transform Based Method onWatermarking Relational Data" International Conference on Multimedia and Ubiquitous Engineering 2008. 
[8] Roberto H. Bamberger, "A Filter Bank for the Directional Decomposition of Images: Theory and Design" IEEE transactions on signal processing. Vol 40, no 4, april 1992.

[9] Farook Sattar, Lars Floreby, G*oran Salomonsson, "Image Enhancement Based ona Nonlinear Multiscale Method" IEEE Transactions On Image Processing, VOL. 6, NO. 6, JUNE 1997.

[10] R.Veluman , V.Seenivasagam "Reversible Blind HF Domain Contourlet Watermarking for Authentication of Digital Images” IJCA Volume 24- No.4, June 2011.

[11] Farhad Rahimi, Hossein Rabbani "A dual adaptive watermarking scheme in contourlet domain for DICOM images" BioMedical Engineering OnLine 2011.
[12] Zhou Wang, A. C. Bovik, H. R. Sheikh, and E. P. Simoncelli, "Image Quality Assessment: From Error Visibility to Structural Similarity" IEEE Transactions On Image Processing, Vol. 13, NO. 4, April 2004.

[13] C.Venkata Narasimhulu, K.Satya Prasad "A New SVD based Hybrid Color Image Watermarking forCopyright Protection using Contourlet Transform" " IJCA Volume 20-No.8, April 2011.

[14] Gonzalez, Richard E Woods "DigitalImageProcessing".

[15] Yaxun Zhou, Wei Jin “A Robust Digital Image Multiwatermarking Scheme in the DWT Domain" ICSAI 2012. 